\title{
El sufijo nominalizador latino -or
}

\author{
Roberto Zamudio Campos \\ Universidad Nacional Mayor de San Marcos \\ magisterdoctus@gmail.com
}

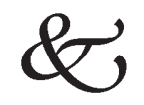

\begin{abstract}
Resumen
En este artículo examinamos una propuesta distinta de nominalización con el sufijo -or con la intención de mostrar una alternativa al proceso derivativo nominal con el sufijo -tor. Este camino comprende no solo un cambio de sufijo sino también un cambio de base derivativa que da mayor solvencia al proceso derivativo.
\end{abstract}

Palabras claves: Derivación nominal, sufijo, tema, supino latino.

\begin{abstract}
In this paper we examine a different proposal for derivation of nouns using the Latin suffix -or in order to show an alternative to the derivational process to form names through the suffix -tor. This alternative way implies not only a change of suffix but also a change in the base from which a word is derived so it provides a better explanation for the derivational process.
\end{abstract}

Keywords: Noun derivation, Suffix, Stem, Latin, Latin supine.

\section{Introducción}

Con esta investigación pretendemos aclarar el panorama de la derivación en latín, por medio del análisis de un sufijo nominalizador. Por ello, la relevancia del estudio radica en la confrontación de las formas propuestas por Menéndez Pidal, asumida durante buen tiempo como válida, la ofrecida por Aída Mendoza que resulta bastante simplificadora, y la que es sostenida actualmente en los estudios históricos.

El artículo está estructurado en cuatro partes. En la primera, presentamos el estado de la cuestión, donde se evidencia la nula dedicación al tema derivativo 
en la enseñanza actual del latín a partir de manuales con el formato tradicional ${ }^{1}$. En la segunda parte, formulamos el problema de nuestra investigación. En la tercera parte, planteamos la hipótesis. En cuarto lugar, presentamos los materiales que nos sirvieron para realizar el trabajo. En la última parte, realizamos el análisis de los datos y damos cuenta del sufijo latino -or, y su interacción en las distintas conjugaciones latinas. Además, señalamos de manera breve cuál es la propuesta actual del sufijo derivativo latino y qué base tiene. Finalmente, planteamos las conclusiones con las cuales refrendamos la hipótesis planteada al inicio.

\section{Estado de la cuestión}

En los distintos libros y manuales de enseñanza de latín, notamos que solo se desarrolla un enfoque exclusivo en temas de tipo morfológico-flexivo y muy poca dedicación a la parte derivativa. Ha sido esta carencia la que en principio nos anima a escribir sobre un problema que se torna mayor cuando por ello, muchas veces, el aprendizaje de la lengua latina es descartado en el aspecto derivacional.

Por lo general, los libros y manuales destinados a la enseñanza del latín utilizados en el Perú se fundan sobre una taxonomía altamente detallada de las distintas categorías gramaticales. Nos nutrimos de antiguos documentos creados en el exterior que repiten el mismo formato o libros elaborados en el país, pero sin ninguna novedad más que su autoría. Un breve recuento de las obras empleadas en la enseñanza del latín en nuestro medio refuerza el panorama acerca de lo expuesto: casi todo es una reproducción de lo mismo.

Los documentos de autoría peruana dedicados al latín no son muchos. Así, tenemos el libro Gramática latina de Dora Bazán (1970), que expone lo más importante a nivel teórico (definiciones, declinaciones y conjugaciones), pero sin ninguna parte práctica ni derivativa. El libro Del latín clásico al romance castellano de Ignacio Ugarte (1979) expone información más detallada y aborda grosso modo la derivación: el último capítulo se dedica al problema de los afijos. Posteriormente, Aída Mendoza publicó Latín básico (1989) y, al alimón con Gertrud Schumacher, Latín jurídico (1993). Ambos libros dan amplia información sobre el aspecto gramatical de la lengua (declinaciones y conjugaciones), pero no toman en cuenta la parte derivativa. Tres años después, María Purificación Estebáñez Gallego publicó Gramática de la lengua latina (1996), en el que ofrece información gramatical morfológica y sintáctica del latín. No obstante, el libro solo expone la naturaleza flexiva de la lengua.

1 Este punto, aunque no es desarrollado in extenso en esta investigación, puede suscitar controversia en la comunidad académica debido a lo restrictivo para el aprendizaje de la lengua latina en la actualidad. 
Nuevamente, Aída Mendoza y Gertrud Schumacher (1997-98) abordan el tema derivativo en el Curso autoinstructivo de latín jurídico, en el que mediante pequeños apartados describen la derivación nominal y verbal de las palabras. Además, con el artículo El sistema de prefijación en latín, Aída Mendoza (1997) se acerca a los prefijos latinos con una explicación detallada de algunos de ellos. Posteriormente, Aída Mendoza y Roberto Zamudio (2005) publicaron Expresiones latinas, el texto pretende ser un antecedente para la lectura de textos en latín y su valor radica en la entrega didáctica de la información en bloques progresivamente complejos.

Los últimos estudios son de Roberto Zamudio quien publicó dos artículos. El primero fue El infijo diminutivo en latín y lexicalización en castellano (2007) y aborda la derivación a través del sufijo diminutivo-apreciativo [-ul-] presente en muchas palabras castellanas pero ya lexicalizado. El segundo artículo titulado El frecuentativo en latín (2008) presenta la derivación de verbos latinos a partir de los participios de otros verbos y la relación que mantienen con sus correspondientes castellanos. In medias res, se advierte fácilmente el escaso estudio de la derivación latina, lo cual nos parece preocupante pues abordar la derivación permitiría enriquecer el panorama morfológico-comparativo entre el latín y el castellano.

\section{Formulación del problema}

Nos llamó la atención que los derivados latinos con el sufijo -tor presentaran ciertas coincidencias formales y semánticas con la base supina de los verbos. La consideración de ello nos planteó la necesidad de responder si -tor es un sufijo nominalizador latino idóneo. Nos interesa estudiar dicho morfema derivativo para establecer precisiones respecto de su verdadera forma latina. Así, el conocimiento de este tema puede establecer un mejor entendimiento respecto de las actuales formas castellanas. Asimismo, tendremos una idea general sobre la formación de palabras en latín pues el procedimiento parece ser recursivo.

\section{Hipótesis}

Luego de observar la derivación latina y el comportamiento de los elementos involucrados en los nombres derivados con la terminación -tor, pensamos que es válido considerar la propuesta de Aída Mendoza respecto del mencionado sufijo. Según Mendoza, la forma del sufijo debiera ser -or puesto que el segmento anterior - $t$ - se encuentra incluido en la base supina ofrecida por la forma verbal que es el punto derivativo. Este planteamiento resulta más 
abarcador pues considera aquellas formaciones que no presentan el segmento -t- en los derivados.

\section{Materiales y métodos}

El corpus de este trabajo está constituido por las palabras extraídas de algunos documentos de carácter lexicográfico como el Diccionario latino-español de Agustín Blánquez Fraile, el Diccionario latino-español de Santiago Segura Munguía, el Diccionario latino-español Vox, el Diccionario de la Real Academia Española y los distintos vocabularios adjuntados en las diferentes gramáticas latinas, como la de Valentí Fiol o José Caratti, que presentan el morfema señalado. La investigación que realizamos es de tipo explicativo-descriptivo ya que exponemos el comportamiento del morfema propuesto por Aída Mendoza y describimos la característica general de los derivados.

Los pasos seguidos parten de la revisión de los distintos documentos que ya mencionamos, a partir de los cuales hemos seleccionado los términos que presentan el sufijo -or. En segundo lugar, atendimos que la existencia del sufijo -or, propio de nombres de agentes como amator o cursor, no conllevara ninguna confusión con el sufijo homógrafo -or, presente en abstractos como amor o timor, adjunto a una base diferente. En tercer lugar, comprobamos que los términos seleccionados correspondan a verbos con formas supinas puesto que ahí radicaría el origen del derivado. Por último, una vez recogida la muestra y salvados los filtros respectivos para la regularidad del corpus, ordenamos el conjunto alfabéticamente y le asignamos el significado respectivo a cada entrada.

\section{Análisis}

\subsection{El sufijo nominalizador -tor con base temática}

Menéndez Pidal (1904:226), en su Manual de gramática histórica española, señala que la derivación de nombres de agente con -tor parte de una base temática: "-tor se une en latín a temas verbales para expresar agente, como acussa-tor, lec-tor, fac-tor".

A continuación, ofrecemos una breve muestra de las formaciones derivadas a partir de una base temática, según el planteamiento de Menéndez Pidal. Cada cuadro contiene los derivados por sufijación con sus respectivas bases y significados para los verbos de las cuatro conjugaciones latinas. El subrayado en los derivados indica la regularidad en la terminación. 
(1)

\begin{tabular}{|c|c|c|c|}
\hline \multicolumn{4}{|c|}{ Primera conjugación } \\
\hline verbo & tema & sustantivo derivado & significado \\
\hline amare & ama- & amator & 'el que ama' \\
\hline clamare & clama- & clamator & 'el que llama' \\
\hline cultare & culta- & cultor & 'el que labra' \\
\hline devorare & devora- & devorator & 'el que devora' \\
\hline
\end{tabular}

(2)

\begin{tabular}{|c|c|c|c|}
\hline \multicolumn{4}{|c|}{ Segunda conjugación } \\
\hline verbo & tema & sustantivo derivado & significado \\
\hline augere & auge- & auctor & 'el que produce' \\
\hline debere & debe- & debitor & 'el que debe' \\
\hline docere & doce- & doctor & 'el que enseña' \\
\hline videre & vide- & visor & 'el que mira' \\
\hline
\end{tabular}

(3)

\begin{tabular}{|c|c|c|c|}
\hline \multicolumn{4}{c|}{ Tercera conjugación } \\
\hline verbo & tema & sustantivo derivado & significado \\
\hline agere & age- & actor & 'el que hace algo' \\
\hline crucifigere & crucifige- & crucifixor & 'el que crucifica' \\
\hline currere & curre- & cursor & 'el que corre' \\
\hline vehere & vehe- & vector & 'el que transporta” \\
\hline
\end{tabular}

(4)

\begin{tabular}{|c|c|c|c|}
\hline \multicolumn{4}{|c|}{ Cuarta conjugación } \\
\hline verbo & tema & sustantivo derivado & significado \\
\hline audire & audi- & auditor & 'el que oye, oyente' \\
\hline salire & sali- & saltor & 'el que da saltos' \\
\hline praeire & praei- & praetor & 'el que va adelante' \\
\hline vincire & vinci- & vinctor & 'el que ata o reúne' \\
\hline
\end{tabular}

Los derivados con -tor que muestran regularidad corresponden a la primera conjugación. Enseguida le suceden los derivados de cuarta y, por último, aquellos de la segunda y tercera conjugación. A excepción de la primera conjuga- 
ción, las demás conjugaciones evidencian derivados que no resultan solo de la simple adjunción de -tor a los temas. Observemos (5):

(5)

\begin{tabular}{|c|c|c|c|c|c|}
\hline verbo & $\begin{array}{c}\text { base } \\
\text { temática }\end{array}$ & + & $\begin{array}{c}\text { sufijo } \\
\text { derivativo }\end{array}$ & $\rightarrow$ & derivado \\
\hline amare & ama- & & & & amator \\
\hline debere & debe- & & \multirow{2}{*}{-tor } & & debitor $^{*}$ \\
\hline currere & curre- & & & & curretor $^{*}$ \\
\cline { 1 - 2 } & & & & auditor \\
\hline
\end{tabular}

En (5), la adjunción del derivativo -tor a los temas de segunda y tercera conjugación no permite derivar los nombres correctos sin alterar sus bases temáticas. Es obvio que hay una reestructuración en la base cuyo elemento final no es la vocal temática como en los agentes de primera y cuarta conjugación. De esta manera, es claro que la adjunción de -tor al tema verbal genera los cambios y produce el derivado. En este proceso, los segmentos involucrados corresponden a los últimos de la base derivativa y al primero del sufijo. Observemos (6).

(6)

\begin{tabular}{|c|c|c|}
\hline verbo & base temática & secuencia derivativa \\
\hline debere & debe- & debetor $>$ debitor \\
\hline currere & curre- & curretor $>$ currtor $>$ curtor $>$ cursor \\
\hline
\end{tabular}

Si estimamos que Menéndez Pidal explica la derivación con -tor hace más de cien años, es lógico pensar que, a raíz de los estudios posteriores en linguiística histórica que hurgan en el sistema del indoeuropeo, la explicación haya encontrado bases más sólidas y coherentes respecto de un fenómeno tan importante ${ }^{2}$. Nos permitimos esta afirmación no solo porque la propuesta de Menéndez Pidal ubica el proceso derivativo en el tema, cuya estructura no siempre alcanza la forma raíz más vocal temática, sino también porque existe otra alternativa verbal que brinda una base regular a los derivados nominales.

\subsection{El supino verbal}

Valentí Fiol (1945) señala que el supino es considerado un sustantivo verbal de tema en - $u$, del que solo quedan dos formas: un acusativo en -um y un dativo en - $u$. No todos los verbos tienen supino, y en la lengua clásica solo unos pocos

2 Sin embargo, actualmente muchos investigadores asumen que la base sigue siendo el tema por la similitud con otras derivaciones latinas. 
poseen la forma en - $u$. Asimismo, el supino en -um se usa como complemento de finalidad con verbos de movimiento y es, en realidad, un acusativo de dirección. Por ejemplo: eo lusum 'voy a jugar', venio salutatum 'voy a saludar'.

El supino se observa al final de una entrada verbal completa como la siguiente:

amo, amas, amare, amavi, amatum (tr): amar

Además, en la formación del supino intervienen el tema verbal (raíz más vocal temática) y el morfema -tum. En (7), observamos la constitución del supino en las entradas verbales de las cuatro conjugaciones y la supresión del morfema flexivo que permitirá obtener la base para posteriores realizaciones.

(7)

\begin{tabular}{|c|c|c|c|c|c|c|c|}
\hline $\begin{array}{l}\text { tema de } \\
\text { presente }\end{array}$ & + & morfema & $\rightarrow$ & supino & $\rightarrow$ & elisión & $\begin{array}{l}\text { base } \\
\text { supina }\end{array}$ \\
\hline ama- & & \multirow{4}{*}{-tum } & & amatum & & amattmm & amat- \\
\hline debe- & & & & debitum & & debittmm & debit- \\
\hline curre- & & & & cursum & & curstmm & curs- \\
\hline audi- & & & & auditum & & audittmm & audit- \\
\hline
\end{tabular}

De esta manera, no solo observamos que el supino puede ofrecer el segmento - $t$ - o -s- como parte de la base derivativa, sino que también posee un contenido activo que puede coincidir con el aportado por un nuevo sufijo, a saber, -or. Otro factor decisivo en esta propuesta se encuentra en que el supino tiene forma de acusativo latino, el cual indica fundamentalmente dirección o fin de una acción verbal. Justamente, los únicos usos del acusativo son la expresión del complemento directo de los verbos transitivos y la de complemento de dirección con verbos de movimiento (Fiol, 1945: 22).

\subsection{El sufijo nominalizador -or con bases supinas}

El sufijo -or es un elemento que ayuda a formar palabras desde los supinos verbales. Las palabras resultantes corresponden a la categoría de nombre, entendido como "el que realiza la acción de X". Gracias a la derivación del supino verbal, los nuevos términos se convierten en realizadores del verbo original: -or es un sufijo deverbal que se añade a la base supina para generar sustantivos agentes.

Aída Mendoza (1998) se ocupó primero de este elemento y se preocupó por definirlo en un opúsculo ${ }^{3}$, al caracterizar el sufijo con la forma -or. Mendoza le

3 El opúsculo corresponde a la publicación hecha bajo el nombre de Curso autoinstructivo de latín jurídico (1998), fasc. 6, p. 7. 
da valor de agente y lo define como 'el que hace la acción'. Asimismo, Valdemar Castells (1997), en una publicación independiente, también cita -or como sufijo derivativo ${ }^{4}$. Ambos autores proponen el sufijo -or en medio de la postura general que postula -tor.

Pensamos que plantear la derivación a partir del tema no es conveniente puesto que la base y el sufijo deben modificarse para la recepción de dos elementos (-tum y -tor) que comparten el mismo segmento -t-. Esto sería innecesario si consideramos la existencia de una base supina que presente el segmento -t- como producto de la adjunción del primer sufijo -tum y reciba solo un nuevo sufijo (-or) sin alteraciones.

Pensamos que es más congruente fundar la modificación de segmentos en una etapa previa (formación del supino) que durante la derivación con -or, pues, además de simplificar ambos procesos (formación del supino y formación del derivado), se libera la base y el sufijo de cualquier cambio.

A continuación, ilustramos la adjunción del sufijo -or a las bases ofrecidas por el supino de cada conjugación latina. En cada una, indicamos los términos formados por sufijación con sus respectivas bases y significados. Además, precisamos que la cantidad de entradas depende de la conjugación que ofrece la posibilidad de derivar agentes con -or a partir de la base supina. Cada término se ubica bajo el rótulo de verbo (en infinitivo), base supina (solo la base), el sustantivo derivado (la palabra derivada) y el significado (castellano).

\section{3.1. Adjunción del sufijo -or a bases supinas de primera conjugación}

Ninguna base supina latina de primera conjugación que permite la adjunción del sufijo -or muestra algún cambio que involucre los elementos de la base como del sufijo en cuestión.

Asimismo, todas las bases supinas de primera conjugación implicadas en el proceso presentan invariablemente, como último elemento, el segmento -t.

(8)

\begin{tabular}{|c|c|c|c|}
\hline \multicolumn{4}{|c|}{ Primera conjugación } \\
\hline verbo & base supina & $\begin{array}{c}\text { sustantivo } \\
\text { derivado }\end{array}$ & significado \\
\hline amare & amat- & amator & 'el que ama' \\
\hline clamare & clamat- & clamator & 'el que vocinglera' \\
\hline cultare & cult- & cultor & 'el que cultiva' \\
\hline devorare & devorat- & devorator & 'el que devora' \\
\hline
\end{tabular}

4 El documento de Castells estuvo publicado en Internet durante un buen tiempo, luego de lo cual fue retirado y vuelto a publicar nuevamente. No encontramos mayores referencias más que las ofrecidas por el mismo documento. 


\subsubsection{Adjunción del sufijo -or a bases supinas de segunda conjugación}

Ninguna base supina de segunda conjugación que permite adjuntar el sufijo -or modifica algún formante en el derivado final. No obstante, las bases supinas de segunda conjugación se distinguen notoriamente de las bases de primera conjugación en su último elemento, pues evidencian la alternancia -t $\sim$-s; esto supone un cambio en los formantes correspondientes a una etapa previa de su formación.

(9)

\begin{tabular}{|c|c|c|c|}
\hline \multicolumn{4}{|c|}{ Segunda conjugación } \\
\hline verbo & base supina & $\begin{array}{c}\text { sustantivo deri- } \\
\text { vado }\end{array}$ & significado \\
\hline augere & auct- & auctor & 'el que produce' \\
\hline debere & debit- & debitor & 'el que debe' \\
\hline docere & doct- & doctor & 'el que enseña' \\
\hline videre & vis- & visor & 'el que mira' \\
\hline
\end{tabular}

\subsubsection{Adjunción del sufijo -or a bases supinas de tercera conjugación}

Ninguna base supina de tercera conjugación que permite lograr la derivación con la adjunción del sufijo -or modifica algún elemento. Por otro lado, debemos señalar que las bases supinas de tercera conjugación se comportan como las de segunda conjugación: sus últimos elementos denotan la alternancia $\mathrm{t}-\sim \mathrm{s}^{-} \sim \mathrm{x}^{-}$, lo cual supone un cambio en una etapa anterior a su formación.

(10)

\begin{tabular}{|c|c|c|c|}
\hline \multicolumn{4}{|c|}{ Tercera conjugación } \\
\hline verbo & base supina & $\begin{array}{c}\text { sustantivo deri- } \\
\text { vado }\end{array}$ & significado \\
\hline agere & act- & actor & 'el que hace algo' \\
\hline crucifixere & crucifix- & crucifixor & 'el que crucifica' \\
\hline currere & curs- & cursor & 'el que corre' \\
\hline vehere & vect- & vector & 'el que transporta” \\
\hline
\end{tabular}

\subsubsection{Adjunción del sufijo -or a bases supinas de cuarta conjugación}

Ninguna base supina de cuarta conjugación que permite la derivación con la adjunción del sufijo -or se modifica. Además de ello, así como en la primera 
conjugación, las bases supinas de verbos de cuarta conjugación muestran regularidad en sus terminaciones pues el elemento final es $-t$.

La diferencia entre derivados de primera y cuarta conjugación es cuantitativa pues el número de derivados agentes que permiten las bases supinas de cuarta conjugación es menor que las producidas a partir de las bases de primera conjugación.

(11)

\begin{tabular}{|c|c|c|c|}
\hline \multicolumn{4}{|c|}{ Cuarta conjugación } \\
\hline verbo & base supina & sustantivo derivado & significado \\
\hline audire & audit- & auditor & 'el que oye' \\
\hline salire & salt- & saltor & 'el que da saltos' \\
\hline praeire & praeit- & praetor & 'el que va adelante' \\
\hline vincire & vinct- & vinctor & 'el que ata o reúne' \\
\hline
\end{tabular}

\subsection{El sufijo nominalizador -tor con bases supinas}

Los estudios actuales de linguiística histórica postulan que los derivados agentes devienen una base supina y el sufijo - tor. ${ }^{5}$ Esto, que parece la integración de la propuesta de Menéndez Pidal, quien defiende -tor, y de quienes postulan una base supina, como Aída Mendoza, es el resultado de los estudios sobre el indoeuropeo. El referente mayor en esta materia es Emile Benveniste (1948), para quien una de las categorías más firmemente establecidas de la morfología nominal indoeuropea es la de nombres de agente en *-t e/r. Sin embargo, los estudios sobre el indoeuropeo no siempre muestran consenso y una posición distinta es la de Guido Gómez Silva (1988), quien señala que el sufijo indoeuropeo *os forma sustantivos (generalmente masculinos) a partir de verbos y significa 'que hace, que ejecuta, que realiza' y que está presente en los nombres defensor, impresor, pastor, relator, traidor:

Ambas posiciones pueden conciliarse si en la explicación del origen de un sufijo se encuentra el otro. De esto, Gertrud Schumacher (2010) señala que el probable que en el origen del nominalizador indoeuropeo *-t $\mathrm{e} / \mathrm{r}$ se encuentre *-os, dado que ambos señalan realizadores de acción. Además, las evidencias respecto de este sistema no llevan a afirmar con seguridad que un elemento pueda descartar a otro pues se trabaja sobre corpus de confiabilidad bastante relativa.

5 David Pharies (2002) señala que la base verbal a la que se adjunta dicho sufijo corresponde a la raíz del supino o participio de perfecto pasivo. 


\section{El sufijo nominalizador -or de nombres abstractos}

Debemos esclarecer la diferencia entre sustantivos derivados de sufijos homófonos. En el caso de -or, notaremos que, además de los sustantivos agentes derivados de supinos como dictator, rector o auditor, existen otros derivados de temas verbales que resultan de la adjunción de -or y que no corresponden a dicho grupo, como amor, favor o valor. La razón estriba en que ambos sufijos son diferentes porque se asientan sobre bases distintas: los derivados agentes son el resultado de adjuntar -or a bases supinas, mientras los derivados abstractos resultan de adjuntar -or a bases temáticas. Hablamos pues de sufijos formalmente iguales con funciones distintas.

No encontramos más definiciones sobre el sufijo -or para nombres abstractos más que la señalada por dos autores: Aída Mendoza, quien menciona que "otros sustantivos terminados igualmente en -or se derivan de raíces verbales simples." ${ }^{\circ}$ y David Pharies (2002), quien señala que -or es un sufijo latino que deriva sustantivos a partir de bases verbales y adjetivas, pero todos los derivados designan nombres abstractos. Enseguida, presentamos algunos derivados en (44).

\begin{tabular}{|c|c|c|c|c|c|c|}
\hline verbo & $\begin{array}{c}\text { base } \\
\text { temática }\end{array}$ & $\begin{array}{c}\text { sufijo } \\
\text { derivativo }\end{array}$ & + & $\rightarrow$ & $\begin{array}{l}\text { sustantivo } \\
\text { derivado }\end{array}$ & significado \\
\hline ardere & ard- & \multirow{8}{*}{-or } & & & ardor & 'calor grande' \\
\hline errare & err- & & & & error & ‘acción equivocada' \\
\hline caelare & cal- & & & & calor & $\begin{array}{c}\text { 'sensación de alta } \\
\text { temp’ }\end{array}$ \\
\hline favere & fav- & & & & favor & ‘ayuda concedida’ \\
\hline terrere & terr- & & & & terror & 'miedo muy intenso' \\
\hline timere & tim- & & & & timor & 'presunción, temor' \\
\hline valere & val- & & & & valor & 'fuerza, actividad' \\
\hline vigere & vig- & & & & vigor & 'actividad notable' \\
\hline
\end{tabular}

En (11) las bases verbales generadoras de los sustantivos abstractos no son actualizadas como supinos, como lo eran los sustantivos agentes. Aunque ambas devienen de verbos, se trata de bases distintas, una supina y otra temática -una base conformada por la raíz verbal, una vez despojada de la vocal temática- por lo cual no se trata del mismo sufijo, sino de sufijos homófonos.

6 Aída Mendoza y Gertrud Schumacher, Curso autoinstructivo de latín jurídico (1997), fasc. 6, p. 9. 
En suma, el sufijo nominalizador -or adjunto a bases supinas deriva nombres agentes, realizadores de la acción de verbos de las cuatro conjugaciones latinas. No es así en el caso de su homófono -or, que a partir de bases temáticas y verbales deriva nombres abstractos. Asimismo, ambos sufijos permiten una derivación heterogénea pues cambian la categoría de la base a la que se adjuntan $(\mathrm{V} \rightarrow \mathrm{N})$.

\section{Conclusiones}

1. El sufijo nominalizador latino -or permite explicar de manera más consistente la derivación. El sustento se encuentra en la concepción de una base supina que permite la simple adjunción del sufijo sin la necesidad de modificar los segmentos involucrados. La alomorfia de base que se dio en latín para la formación del supino ofrece una base supina como punto derivativo.

2. Los derivados latinos con el sufijo -or que no corresponden a realizadores de la acción derivan de bases no supinas. Estos sustantivos derivan de bases temáticas y denotan nombres abstractos. A pesar de que las bases supinas y las bases temáticas son ofrecidas por el verbo, -or debe considerarse un sufijo distinto. Así, del verbo amare se obtiene el agente amator 'el que ama' y el abstracto amor 'sentimiento de afecto' derivados respectivos de la base supina amat- y la base temática am-. Por lo tanto, es imposible que se trate del mismo sufijo. Este proceso es recurrente en aquellos pares que presentan la misma terminación y derivan del mismo verbo, como errator y error o territor y terror.

3. Finalmente, en su paso al castellano, la forma de los sustantivos latinos agentes que presentan el sufijo -or no sufrió un cambio importante en la mayoría de casos. Salvo las sonorizaciones de $t>d$ las palabras son las mismas. Respecto del aspecto semántico, el significado primigenio de los derivados latinos designa al agente de la acción (litigator 'el que litiga', abolitor 'el que abole', rector 'el que dirige'); mientras que las formas castellanas, además del agente, producen nuevas entradas con sentidos diversos: locativo (mirador), instrumental (destornillador) o accesorio (recogedor).

\section{Referencias bibliográficas}

ALVAR, Manuel (2006). La formación de palabras en español. Madrid: Arco Libros. BAZÁN, Dora (1971). Gramática latina. Lima: UNMSM.

BLÁNQUEZ, Agustín (1954). Diccionario latino-español. España: Ramón Sopena S. A.

CATALANO, Pierangelo (1990). El digesto de Justiniano, Libro I. Lima: PUCP.

COROMINAS, Joan (1961). Breve diccionario etimológico de la lengua castellana. Madrid: Gredos. 
ESTEBÁÑNEZ, María (1995). Gramática de la lengua latina. Lima: PUCP.

FELÍU, Elena (2003). Morfología derivativa y semántica léxica: la prefijación de auto-, co- e inter-. Madrid: Universidad Autónoma de Madrid.

FIOL, Valentí (1993). Gramática de la lengua latina. Madrid: Bosch S. A.

FIOL, Valenti (1993). Sintaxis latina. Madrid: Bosch S. A.

GÓMEZ, Guido (1998). Breve diccionario etimológico de la lengua española. México: Fondo de Cultura Económica.

LLOYD, Paul (1993). Del latín al español. Madrid: Gredos.

LORENZO, Guillermo (1998). Curso de morfología generativa. Lima: Universidad Federico Villarreal.

LORENZO, Guillermo (1997).The concise Oxford dictionary of linguistics. Oxford: Oxford University Press.

MENDOZA, Aída (1989). Latín básico. Curso universitario (mimeografiado). Lima: UNMSM. MENDOZA, Aída y Gertrud SCHUMACHER (1993). Latín jurídico. Lima: Unión Latina.

MENDOZA, Aída y Gertrud SCHUMACHER (1997). Curso autoinstructivo de latín jurídico. Lima: INVEL.

MENDOZA, Aída y Roberto ZAMUDIO (2005). Expresiones latinas. Lima: INVEL.

MENDOZA, Aída y Roberto ZAMUDIO (2005). "Nombres propios de procedencia latina” en Escritura y Pensamiento, Año VIII, № 17, 2005; pp.153-182.

MENÉNDEZ Pidal, R (1968). Manual de Gramática Histórica Española. Madrid: Espasa Calpe S. A.

PENA, Jesús (2008). "El cambio morfológico en el interior de las series de derivación” en Revista de Investigación Lingüística, $\mathrm{N}^{\circ} 11$; pp. 233-248.

PHARIES, David (2002). Diccionario etimológico de sufijos españoles y de otros elementos. Madrid: Ed. Gredos.

REAL ACADEMIA ESPAÑOLA (2001). Diccionario de la Real Academia Española. Madrid: Espasa Calpe.

REAL ACADEMIA ESPAÑOLA (2009). Nueva gramática de la lengua española. Madrid: Espasa Calpe.

REBOLLO, Miguel (1997). "Precisiones sobre los llamados temas" en Anuario de estudios filológicos. 20; pp.

SEGURA, Santiago (2007). Diccionario por raíces del latín y de las voces derivadas. Deusto: Universidad de Deusto.

UGARTE, Ignacio (1979). Del latín clásico al romance castellano. Lima: Facultad de Teología Pontificia y Civil de Lima.

VARELA, Soledad (1990). Fundamentos de morfología. Madrid: Síntesis.

VARELA, Soledad (2005). Morfología léxica: la formación de palabras. Madrid: Gredos.

ZAMUDIO, Roberto. "El infijo diminutivo en latín y lexicalización en castellano" en Escritura y Pensamiento Año X, N.o 20, 2007; 21-46.

ZAMUDIO, Roberto. “El frecuentativo en latín” en Escritura y Pensamiento. Año XI, N. 23, 2008; pp.163-187. 